\title{
Comportamiento microclimático diurno, en temporada seca, de tres estructuras para agricultura protegida en el trópico seco
}

\author{
Edwin Andres Villagran ${ }^{1}(D)$, Jorge Eliecer Jaramillo ${ }^{2}$, Rommel Igor León Pacheco ${ }^{3}[$, \& Roberto Ramirez \\ Matarrita4
}

1. Corporación Colombiana de Investigación Agropecuaria - AGROSAVIA, Centro de Investigación Tibaitata, Mosquera, Cundinamarca, Colombia; evillagran@agrosavia.co

2. Corporación Colombiana de Investigación Agropecuaria - AGROSAVIA, Centro de Investigación La Selva, Rionegro, Antioquia, Colombia; jejaramillo@agrosavia.co

3. Corporación Colombiana de Investigación Agropecuaria - AGROSAVIA, Centro de Investigación Caribia, Sevilla, Zona Bananera, Magdalena, Colombia; rleon@agrosavia.co

4. Instituto Nacional de Innovación y Transferencia en Tecnología Agropecuaria de Costa Rica - INTA, Estación Experimental Enrique Jiménez Núñez, Cañas, Guanacaste, Costa Rica; rramirez@inta.go.cr

\author{
Recibido 30-III-2020 • Corregido 24-VII-2020 • Aceptado 21-IX-2020 \\ DOI: https://doi.org/10.22458/urj.v12i2.2854
}

\begin{abstract}
Diurnal microclimatic behavior, during the dry season, of three structures for protected agriculture in the dry tropics". Introduction: In Costa Rica, the use of protected agricultural structures for horticultural production has increased in recent years, although there is little information on their microclimatic behavior. Objective: Our objective was to evaluate the behavior of airflow patterns and their effect on the spatial distribution of temperature and relative humidity inside three types of protected agricultural structure designed for family farming systems. Methods: The study was done in Guanacaste, Costa Rica, in the dry season of 2019, with a computational fluid dynamics model for the development of nine steady state simulations. The 3D model was experimentally validated by collecting climate information in each of the structure prototypes. Results: For the three structures, the goodness-of-fit parameters between measured and simulated data showed mean absolute error and root mean square error values of 0,21 $0,44^{\circ} \mathrm{C}$ and $1,65-3,40 \%$ relative humidity. Measured and simulated data had the same trends in the showed; the air flow patterns inside the structures were highly dependent on external wind speed and direction. The temperature and relative humidity conditions inside the three structures had a considerably homogeneous behavior. Conclusions: In the three small protected agricultural structures tested, which are usually used in family agriculture, no significant differences were found for thermal and hygrometric behavior inside each of the structures under these testing conditions.
\end{abstract}

Keywords: Airflow pattern, temperature, relative humidity, family farming, simulation.
RESUMEN. Introducción: En Costa Rica, el uso de estructuras agrícolas protegidas para la producción hortícola se ha incrementado en los últimos años, aunque existe poca información sobre su comportamiento microclimático. Objetivo: Nuestro objetivo fue evaluar el comportamiento de los patrones de flujo de aire y su efecto en la distribución espacial de la temperatura y la humedad relativa dentro de tres tipos de estructuras agrícolas protegidas diseñadas para sistemas agrícolas familiares. Métodos: El estudio se realizó en Guanacaste, Costa Rica, en la época seca de 2019, con un modelo de dinámica de fluidos computacional para el desarrollo de nueve simulaciones de estado estacionario. El modelo 3D se validó experimentalmente mediante la recopilación de información climática en cada uno de los prototipos de estructura. Resultados: Para las tres estructuras, los parámetros de bondad de ajuste entre datos medidos y simulados tuvieron error absoluto medio y error cuadrático medio de $0,21-0,44^{\circ} \mathrm{C}$ y $1,65-3,40 \%$ de humedad relativa. Los datos medidos y simulados tuvieron las mismas tendencias; los patrones de flujo de aire dentro de las estructuras dependieron en gran medida de la velocidad y dirección del viento externo. Las condiciones de temperatura y humedad relativa dentro de las tres estructuras tuvieron un comportamiento considerablemente homogéneo. Conclusiones: En las tres pequeñas estructuras agrícolas que probamos, usadas habitualmente en agricultura familiar, no se encontraron diferencias significativas del comportamiento térmico e higrométrico interno en estas condiciones de prueba.

Palabras clave: Patrón de flujo de aire, temperatura, humedad relativa, agricultura familiar, simulación. 
La producción hortícola en Costa Rica se realiza principalmente en el valle central y bajo un enfoque de producción a campo abierto, lo que implica que existe una alta vulnerabilidad de los cultivos al ataque de plagas y enfermedades y a condiciones climáticas extremas (Ramírez-Vargas \& Nienhuis, 2012). Esta problemática en países tropicales se puede solucionar mediante el uso de estructuras de agricultura protegida, dentro de las que se incluyen; casas de malla, invernaderos, macro y micro túneles, entre otras. En la última década a nivel local la implementación de este tipo de estructuras para la producción agrícola a nivel de pequeño y mediano productor ha tenido un aumento relevante pasando de 688 a 5 900ha (Rojas Rishor, 2015; MAG, 2018). Una de las características principales del tipo de estructuras utilizado es su bajo costo y bajo nivel tecnológico, por lo tanto la gestión del microclima se limita a la eficiencia de la ventilación natural y a otro tipo de estrategias de climatización pasiva como lo es el uso de mallas de sombrío o blanqueamiento de cubiertas con el fin de reducir el grado de transmisión de radiación solar desde la zona cubierta.

La ventilación natural depende de dos fuerzas impulsoras: la convección forzada o ventilación dinámica causada por la acción del viento exterior y la convección libre o ventilación térmica vía flotabilidad causada por la diferencia de temperaturas entre el exterior y el interior de la estructura agrícola evaluada (Espinoza et al., 2017). La ventilación afecta la temperatura interna del invernadero de forma tal que en horas de alta radiación se hace necesario circular aire del exterior hacia el interior del invernadero en forma homogénea con el fin de controlar los excesos de temperatura. Este movimiento del flujo de aire debe permitir el intercambio de calor y masa entre las plantas del cultivo y el aire circulante en el invernadero (He et al., 2017). Adicionalmente, la ventilación natural es la encargada de controlar excesos de humedad y mantener los niveles de $\mathrm{CO}_{2}$ en rangos cercanos al nivel presente en la atmósfera con el fin de no limitar el proceso de fotosíntesis en las plantas (Molina-Aiz et al., 2017; Villagrán \& Bojacá, 2019a).

La ventilación natural es altamente dependiente de factores meteorológicos como la velocidad, intensidad y dirección del viento exterior, factores arquitectónicos como; la geometría y forma de la cubierta, el área y volumen de la estructura, el tamaño y ubicación de las superficies ventilación y el uso de mallas anti-insecto. De acuerdo a lo anterior, este método de climatización puede considerarse fluctuante y en ocasiones ineficiente para realizar una adecuada gestión del microclima generado en el interior de invernaderos o cualquier tipo de estructuras de agricultura protegida (McCartney \& Lefsrud, 2018). Una de las técnicas de modelado y simulación más importantes y ampliamente aplicadas al estudio aerodinámico y micro climático en estructuras de agricultura protegida ha sido la dinámica de fluidos computacional (CFD) que actualmente es conocida como una técnica robusta y avanzada para el diseño en muchos campos de la ingeniería (Tong, Christopher, \& Zhang, 2018; Tong \& Christopher, 2018). El uso de CFD ha contribuido a mejorar u optimizar los sistemas de ventilación en invernaderos o casas de malla en países como España (Molina-Aiz, Valera, Peña, Gil, \& López, 2009), México (Flores-Velázquez, Mejía-Sáenz, Montero-Camacho, \& Rojano, 2011) y Colombia (Villagrán, Gil, Acuña, \& Bojacá, 2012; Villagrán, Baeza-Romero, \& Bojacá, 2019).

En esta investigación se utilizó un enfoque experimental que contemplo la implementación y validación de un modelo numérico de simulación CFD-3D, con el objetivo de estudiar los flujos de aire y su efecto sobre las distribuciones de la temperatura y humedad relativa en el interior de tres estructuras de agricultura protegida diseñadas para la producción agrícola bajo el enfoque de agricultura familiar en la provincia de Guanacaste-Costa Rica. 


\section{MATERIALES Y MÉTODOS}

Descripción del invernadero y registro de condiciones climáticas: El estudio experimental se desarrolló en tres estructuras de agricultura protegida: un modelo de casa malla tipo 1 (SHM1) de dimensiones $14 \mathrm{~m}$ de largo, $11 \mathrm{~m}$ de ancho y $3 \mathrm{~m}$ de alto, un modelo de casa malla tipo 2 (SHM2) de dimensiones $14 \mathrm{~m}$ de largo, $13 \mathrm{~m}$ de ancho y $5 \mathrm{~m}$ de alto estos dos modelos de casa malla usaron la misma referencia comercial de malla anti-insectos, por último se evaluó un invernadero tipo túnel (MG) cubierta de plástico y con áreas laterales y cenitales cubiertas con malla anti-insectos, las dimensiones de MG fueron $14 \mathrm{~m}$ de largo, $7 \mathrm{~m}$ de ancho y $8 \mathrm{~m}$ de alto (Fig. 1).

Las tres estructuras evaluadas se establecieron en La Estación Experimental Enrique Jiménez Núñez, del Instituto Nacional de Innovación y Transferencia en Tecnología Agropecuaria (INTA), ubicados en Cañas, Guanacaste, Costa Rica. Las condiciones meteorológicas dominantes para las horas del periodo diurno entre las 8 y las 16 horas del día tales como; temperatura, humedad relativa, radiación global, dirección y velocidad del viento fueron registradas y almacenadas durante un periodo de 60 días de la temporada seca comprendidos entre el 01 de noviembre y el 31 de diciembre de 2019 y con una frecuencia de medición de 10 minutos. Para ello se utilizó una estación meteorológica Vantage Pro2 (Davis Instruments, Hayward, California, USA CA). La temporada seca fue seleccionada para realizar el ensayo por ser un periodo de tiempo donde se suelen presentar limitantes ambientales para la producción agrícola tales como; niveles supra-optimos de radiación solar con elevados valores de temperatura del aire.

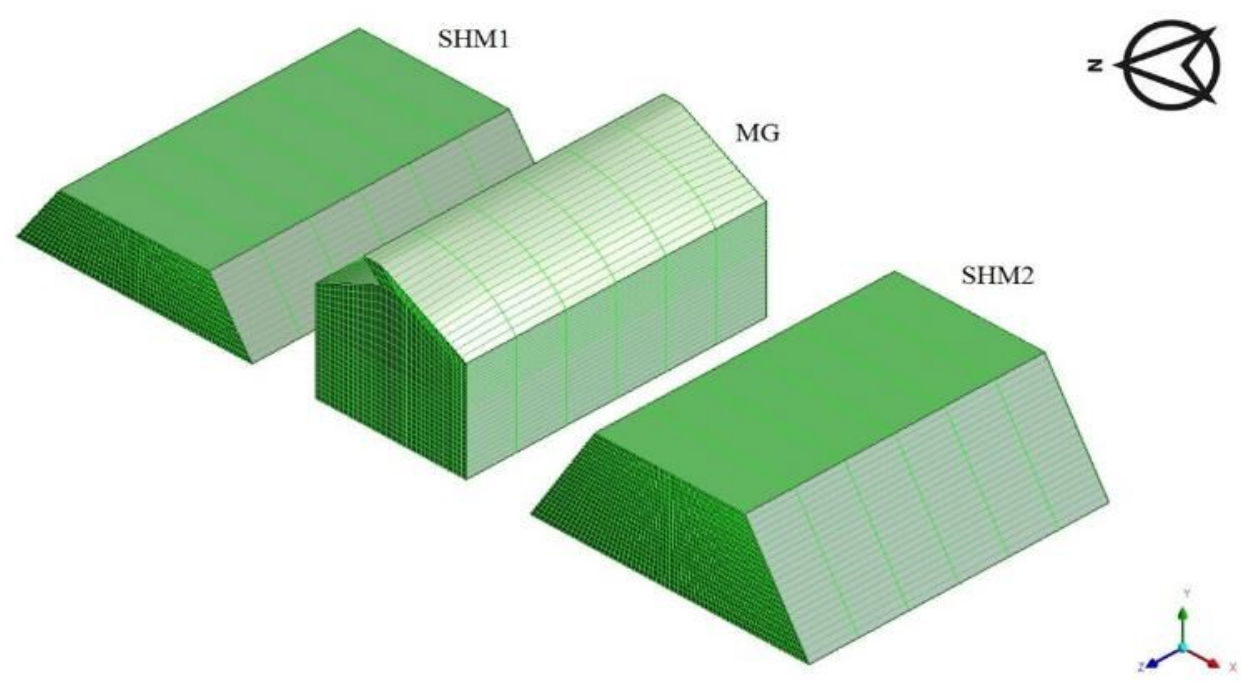

Fig. 1. Vista isométrica de las 3 estructuras evaluadas.

Modelo numérico: El movimiento y la trasferencia de calor de un fluido en movimiento en el interior de una estructura de agricultura protegida puede ser descrito matemáticamente mediante las ecuaciones de Navier-Stokes:

$$
\nabla(\rho \phi \vec{v})=\nabla(\Gamma \nabla \phi)+S \phi
$$

Donde, $v \overrightarrow{ }$ es el vector de velocidad $\left(\mathrm{m} / \mathrm{s}^{-1}\right)$, $\Gamma$ es el coeficiente de difusión $\left(\mathrm{m}^{2} / \mathrm{s}^{-1}\right), \rho$ es la densidad del fluido $\left(\mathrm{kg} / \mathrm{m}^{-3}\right), \nabla$ es el operador nabla, $\varphi$ representa la variable de concentración y $\mathrm{S}$ representa el término fuente (Piscia, Muñoz, Panadès, \& Montero, 2015). La ecuación anterior es 
resuelta mediante el método de volúmenes finitos para la condición de estado estable y en cada volumen de control ( $\mathrm{V}$ ) su forma integral se representa así:

$$
\int_{V}^{X} \nabla \cdot(\rho \phi \vec{v}) d V=\int_{V}^{X} \nabla \cdot(\Gamma \nabla \phi) d V+\int_{V}^{X} \mathrm{~s} \phi d V
$$

La naturaleza turbulenta del flujo de aire se simuló utilizando el modelo de turbulencia estándar $k-\varepsilon$ con funciones de pared estándar, este modelo ha sido ampliamente usado y validado en estudios de ventilación natural, los resultados obtenidos de su uso ha mostrado ser bastante confiables en el proceso computacional (Teitel \& Wenger, 2014). Los efectos de flotabilidad influenciados por el cambio de densidad del aire estarán presentes (Espinal-Montes, Lorenzo LópezCruz, Rojano-Aguilar, Romantchik-Kriuchova, \& Ramírez-Arias, 2015), los cuales pueden ser modelados a través del modelo de Bousinessq, que se describe mediante la siguiente expresión:

$$
\left(\rho-\rho_{0}\right) g=-\rho_{0} \beta\left(\mathrm{T}-\mathrm{T}_{0}\right) \mathrm{g}
$$

Donde $\beta$ corresponde al coeficiente de expansión térmica del aire $\left({ }^{\circ} \mathrm{C}\right), T$ a la temperatura en el instante analizado $\left({ }^{\circ} \mathrm{C}\right), \rho_{0}$ y $T_{0}$ a los valores de referencia de la densidad $\left(\mathrm{Kg} / \mathrm{m}^{-3}\right)$ y la temperatura $\left({ }^{\circ} \mathrm{C}\right)$ respectivamente, esta aproximación se realizan cuando los diferenciales térmicos en el dominio computacional son inferiores a $30^{\circ} \mathrm{C}$, asi mismo cuando los diferenciales de la densidad son requeridos unicamente por el término de flotación de la ecuación actual y existe una relación lineal entre la temperatura, la densidad, y todas las demás propiedades extensivas de los fluidos son constantes (Norton Sun, Grant, Fallon, \& Dodd, 2007; Kim et al., 2008). El modelo de radiación seleccionado fue el de ordenadas discretas (DO) con discretización angular, este permite agregar la radiación como un término fuente en la ecuación de energía para ser modelada en medios semitransparentes como es el caso de los plásticos de las cubiertas de invernadero y las mallas a pruebas de insectos (Bartzanas, Katsoulas, \& Kittas, 2012).

El método de solución semi implícito para la ecuación de presión-velocidad (SIMPLE) fue aplicado para resolver el campo de flujo del fluido simulado, con esquemas de discretización de segundo orden, por otro lado los criterios de convergencia del modelo fueron establecidos con valores para los residuales de $10^{-6}$ para la ecuación de energía y $10^{-4}$ para las ecuaciones de continuidad, momento y turbulencia, valores que han demostrado ser precisos en estudios similares (Saberian \& Sajadiye, 2019).

Dominio computacional y condiciones de simulación iniciales: La construcción del dominio computacional y el proceso de mallado de dicho dominio se realizó mediante la utilización del software comercial de preprocesamiento ANSYS-ICEM (v.18.2). Este dominio se contruyó siguiendo las recomendaciones incluidas en el estudio de efecto eólico de Tominaga et al. (2008) y aplicadas con éxito en estudios numéricos como el de Perén, van Hooff, Leite, y Blocken (2016). Las dimensiones del dominio seleccionadas fueron de $60 \mathrm{~m}$ de alto, $250 \mathrm{~m}$ de ancho y $214 \mathrm{~m}$ de largo. Posteriormente, el dominio computacional dividió en una malla no estructurada con resoluciones más finas impuestas cerca del suelo, el techo y las paredes de cada una de las estructuras evaluadas, esto porque en estas regiones los gradientes térmicos pueden ser más pronunciados.

La malla se configuró con elementos cuadrados compuesta por un total de volúmenes discretizados en el espacio de 146139, 202234 y 203654 para SHM1, SHM2 Y MG, respectivamente y de 4534267 volúmenes para todo el dominio computacional, estos tamaños fueron definidos una vez se realizo el test de independencia de malla, el cual, consistio en evaluar nueve mallas de tamaño 
diferente al seleccionado, este test de independencia se realizo siguiendo el procedimiento recomendado por Villagrán et al. (2019). La calidad de la malla fue evaluada mediante el determinante relativo $3 \times 3$, el cual mostró un comportamiento superior al $94,2 \%$ con celdas entre 0,95 y 1 lo que indica que un alto porcentaje de los elementos de malla son perfectamente regulares (Ansys, 2016).

En la parte inferior del dominio computacional se le impuso la condición de pared al igual que a la cubierta del invernadero, en función del escenario a la zona de entrada de aire se le impuso una condición de velocidad de entrada con un perfil logarítmico de velocidad, creando una función definida por el usuario con la siguiente relación:

$$
V_{2}=V_{1} \frac{\left(\frac{h_{2}}{z_{0}}\right)}{\left(\frac{h_{1}}{z_{0}}\right)}
$$

Donde $V_{1}$ es la velocidad de viento medida en una altura $h_{1} ; V_{2}$ es la velocidad del viento calculada para una altura $h_{2}$ y $z_{0}$ es la longitud del coeficiente de rugosidad expresado en metros, valor que está en función del tipo de superficie de las áreas vecinas a las estructuras de agricultura protegida, para este caso se considero un valor de 0,1 que es propuesto para tierras de cultivo con algunas cortinas rompe vientos a más de $1 \mathrm{~km}$ de distancia (Bañuelos-Ruedas, Ángeles-Camacho, \& Rios-Marcuello, 2010). Para la zona de salida de aire se le impuso una condición de salida de presión, los otros lados del dominio computacional fueron establecidos con una condición de propiedad simétrica, las propiedades físicas y ópticas de los materiales para SHM1, SHM2 Y MG se establecieron a partir de los estudios de Villagrán et al. (2019); Villagrán, Ramirez, Rodriguez, Pacheco, y Jaramillo (2020) y Flores-Velazquez, Ojeda, Villarreal-Guerrero, y Rojano, (2017). Estas propiedades fueron usadas en cada una de las simulaciones realizadas (Cuadro 1).

\section{CUADRO 1}

Propiedades ópticas y físicas de los materiales incluidos en el dominio computacional.

\begin{tabular}{llll}
\hline & Aire & Suelo & Polietileno \\
\hline Densidad $\left(\rho, \mathrm{kg} \mathrm{m}^{-3}\right)$ & 1,225 & 1300 & 923 \\
Conductividad térmica $\left(\mathrm{k}, \mathrm{W} \mathrm{m}^{-1} \mathrm{~K}^{-1}\right)$ & 0,0242 & 1,5 & 0,38 \\
Calor especifico $\left(\mathrm{C} p, \mathrm{~J} \mathrm{~K}^{-1} \mathrm{~kg}^{-1}\right)$ & 1006,43 & 800 & 2300 \\
Coeficiente de expansión térmica $\left(\mathrm{K}^{-1}\right)$ & 0,0033 & & \\
Absorción & 0,19 & 0,9 & 0,37 \\
Coeficiente de dispersión & 0 & 0,12 & 0 \\
Índice de refracción & 1 & 1,92 & 1,77 \\
Emisividad & 0,9 & 0,95 & 0,7 \\
\hline & Permeabilidad de la cara $(\alpha)=2,86 \mathrm{e}^{-9} \mathrm{y}$ \\
Tratamiento de medio poroso & coeficiente de arrastre $\left(\mathrm{C}_{2}\right) 11131$, \\
& Espesor medio poroso 0,000372 \\
\hline
\end{tabular}

Calibración del modelo CFD y escenarios simulados: En el interior de SHM1, SHM2 Y MG sobre la sección media longitudinal (eje Z) en un plano transversal (eje X), a una altura de a 1,6m sobre el nivel del suelo (eje Y), se distribuyeron de manera uniforme y protegidos de la radiación directa, tres sensores tipo data-logger $\mathrm{HOBO}^{\circledR}$ Pro RH-Temp H08-032-08 (Onset Computer Corp., Pocasset, USA), los cuales, se utilizaron para registrar y almacenar los valores de temperatura $\left({ }^{\circ} \mathrm{C}\right)$ y humedad relativa (\%). Al igual que en el ambiente exterior la frecuencia de medición y el registro de datos fue cada 10 minutos. 
Los datos meteorológicos externos obtenidos durante el periodo de medición fueron analizados, seleccionando de manera aleatoria los datos de la hora 13 (Cuadro 2). Estos se impusieron como condiciones de entrada para la simulación de validación; una vez culminada la simulación se realizó el postproceso extrayendo los datos de temperatura y humedad relativa del aire interior en los puntos que coincidían con los puntos internos de muestreo, los datos obtenidos por medio de simulación y experimentación fueron comparados a través del cálculo del error medio absoluto (MAE) y la raíz del error cuadrático medio (RMSE).

$$
\begin{aligned}
& \mathrm{MAE}=\frac{1}{\mathrm{~m}} \sum_{\mathrm{i}=1}^{\mathrm{m}}|\mathrm{Dmi}-\mathrm{Dsi}| \\
& \text { RMSE }=\sqrt{\frac{\left.\sum_{\mathrm{i}=1}^{\mathrm{m}}|\mathrm{Dmi}-\mathrm{Dsi}|^{2}\right)}{\mathrm{m}}}
\end{aligned}
$$

Donde Dmi y Dsi, son los valores de los datos observados y el valor del dato simulado, respectivamente y $\mathrm{m}$ el número de datos comparados. Una vez validado el modelo CFD, éste se usará para la evaluación del comportamiento micro climático de SHM1, SHM2 Y MG estableciendo como condiciones de entrada al modelo CFD, el valor de los parámetros meteorológicos medios obtenidos para cada una de las horas evaluadas (Cuadro 2).

\section{CUADRO 2}

Condiciones meteorológicas usadas como condiciones iniciales de simulación.

\begin{tabular}{cccccc}
\hline Hora & $\begin{array}{c}\text { Temperatura } \\
\left({ }^{\circ} \mathbf{C}\right)\end{array}$ & $\begin{array}{c}\text { Velocidad } \\
\text { del viento } \\
(\mathbf{m} / \mathbf{s})\end{array}$ & $\begin{array}{c}\text { Dirección } \\
\text { del viento }\end{array}$ & $\begin{array}{c}\text { Humedad } \\
\text { relativa } \\
(\%)\end{array}$ & $\begin{array}{c}\text { Radiación } \\
\text { solar } \\
\left(\mathbf{w} / \mathbf{m}^{\mathbf{2}}\right)\end{array}$ \\
\hline 8 & 27,9 & 2,07 & $\mathrm{~N}$ & 70 & 345 \\
9 & 28,1 & 2,67 & $\mathrm{~N}$ & 68 & 435 \\
10 & 29,4 & 2,81 & $\mathrm{NE}$ & 65 & 514 \\
11 & 30,5 & 3,14 & NNW & 61 & 601 \\
12 & 32,4 & 3,70 & $\mathrm{NE}$ & 57 & 780 \\
13 & 33,1 & 2,61 & NNW & 55 & 791 \\
14 & 32,6 & 2,34 & NE & 58 & 659 \\
15 & 31,8 & 2,22 & SSE & 62 & 582 \\
16 & 30,9 & 2,01 & SSE & 64 & 508 \\
\hline
\end{tabular}

\section{RESULTADOS}

Validación del modelo CFD: Los valores de los parámetros de bondad de ajuste MAE y RMSE calculados para la temperatura y humedad relativa entre los valores medidos y simulados mediante CFD para la temperatura en SHM1, SHM2 y MG presentaron valores entre 0,20 y $0,44^{\circ} \mathrm{C}$, mientras que para la humedad relativa variaron entre 1,65 y 3,40\%, respectivamente (Cuadro 3 ).

Por otro lado, se realizó la comparación gráfica de las tendencias de los sets de datos medidos y simulados para los puntos de muestreo de cada una de las estructuras. Las gráficas obtenidas mostraron una tendencia de comportamiento similar para la temperatura y la humedad relativa en el ancho de cada una de las estructuras evaluadas (Fig. 2).

Patrones de flujo de aire: Para las 8 y 12 horas se puede observar cómo los vectores de velocidad muestran una reducción respecto a la velocidad del ambiente exterior, esto sucede una vez el flujo de aire impacta sobre el costado de barlovento de la estructura SHM2. Los valores medios de velocidad de los flujos de aire fueron de 1,34 y $1,92 \mathrm{~m} / \mathrm{s}$ para estas horas, lo que representa una reducción del flujo de aire respecto a la velocidad del viento en el exterior de 64,2 y $51,8 \%$, respectivamente. Estos flujos de aire en el interior de SHM2 mostraron un comportamiento 
con dos direcciones dominantes, una en sentido horizontal hacia el costado de sotavento y otra en sentido vertical dirigida hacia la zona de la cubierta (Fig. 3a, 3b).

\section{CUADRO 3}

Parametros de bondad de ajuste del modelo CFD.

\begin{tabular}{ccccc}
\hline & \multicolumn{2}{c}{ Temperatura $\left({ }^{\circ} \mathrm{C}\right)$} & \multicolumn{2}{c}{ Humedad Relativa (\%) } \\
Estructura & MAE (\%) & RMSE (\%) & MAE (\%) & RMSE (\%) \\
\hline SHM1 & 0,41 & 0,44 & 3,30 & 3,40 \\
SHM2 & 0,26 & 0,27 & 2,20 & 2,40 \\
MG & 0,20 & 0,21 & 1,65 & 1,90 \\
\hline
\end{tabular}
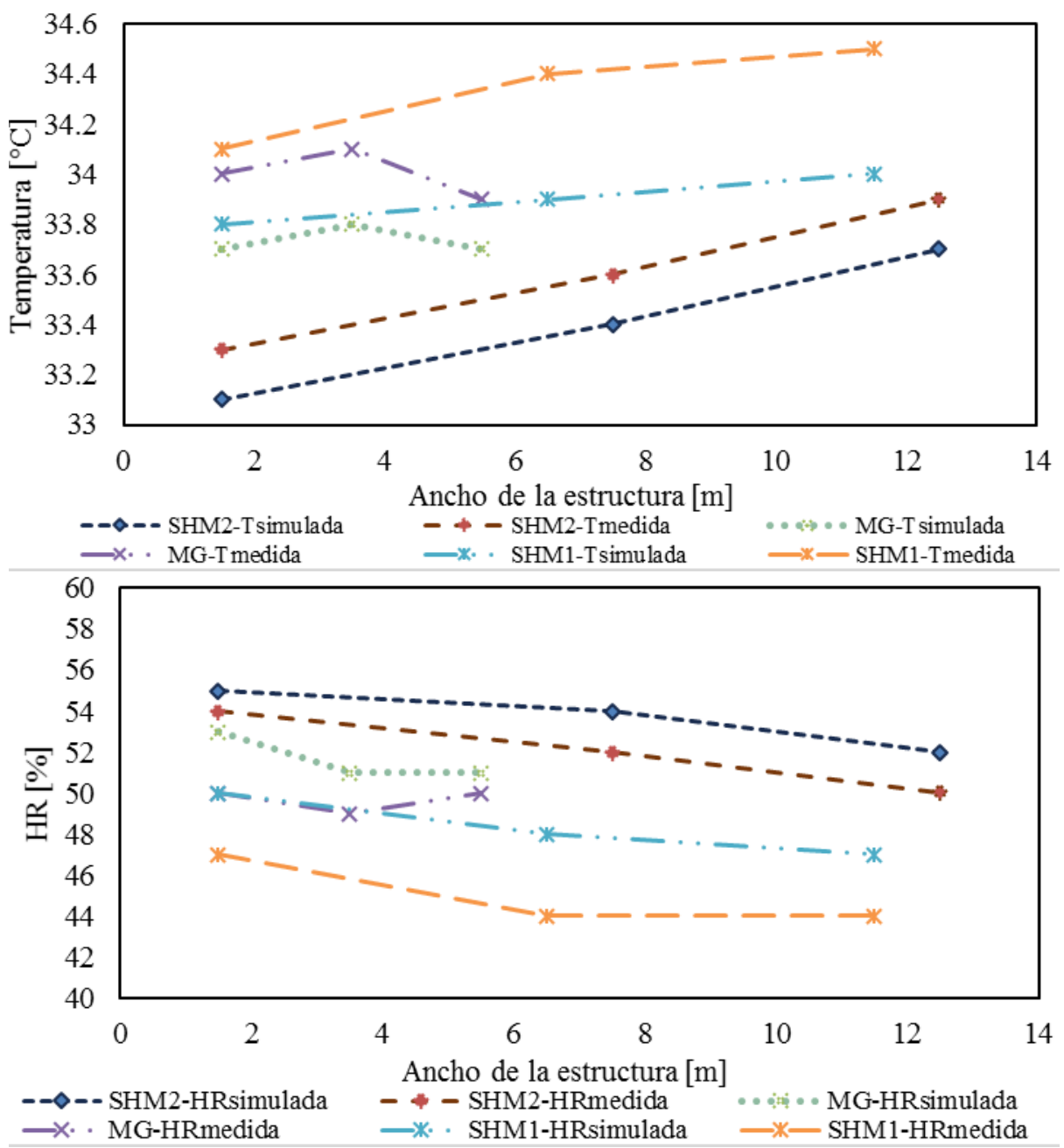

Fig. 2. Perfiles de temperatura $\left({ }^{\circ} \mathrm{C}\right)$ y humedad relativa (\%) simulados y medidos en $\mathrm{SHM} 1, \mathrm{SHM} 2$ y $\mathrm{MG}$.

Por otro lado, el campo de flujo de aire para estas horas en MG y SHM1 mostraron un comportamiento similar, donde cada uno de los patrones de flujos de aire ingresó a las estructuras por los costados de barlovento generando una célula convectiva que se mueve entre la cubierta, el costado de sotavento y el suelo, los valores medios de velocidad de los patrones de flujo de aire fueron de 0,97 y $0,87 \mathrm{~m} / \mathrm{s}$ en SHM1 y MG para la hora 8 , mientras que, para la hora 12 el valor medio de la velocidad fueron de 1,10 y $1,01 \mathrm{~m} / \mathrm{s}$ en SHM1 y MG, respectivamente. La reducción de la 
velocidad del aire respecto al exterior en estos casos fue de 42,1 y $46,8 \%$ para la hora 8 y 27,8 y 27,3\% para la hora 12 en SHM1 Y MG (Fig. 3a, 3b).
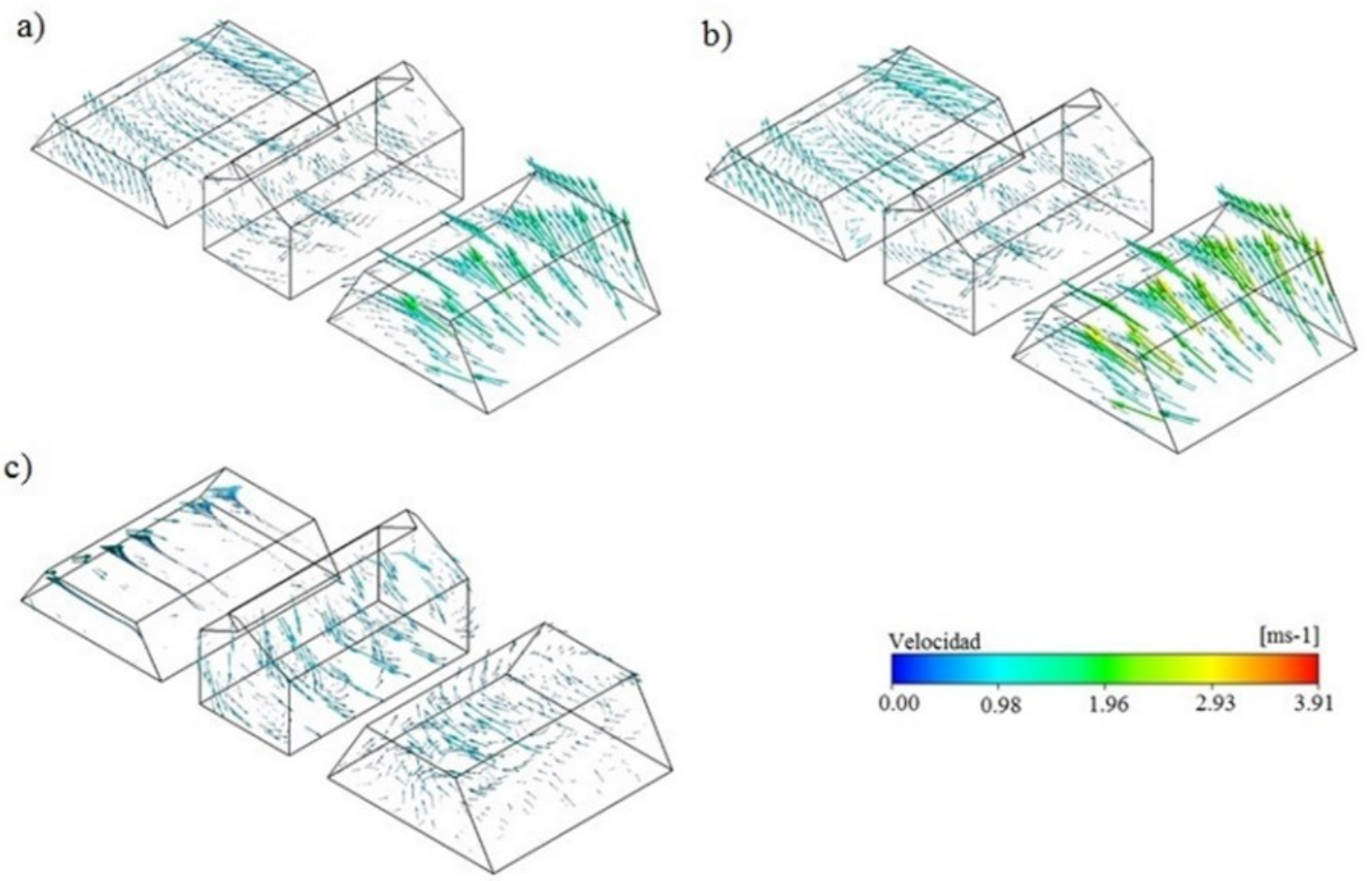

Fig. 3. Patrones de flujo de aire $\left(\mathrm{ms}^{-1}\right)$ simulados (a) Hora 8, (b) Hora 12 y (c) Hora 16.

Para la hora 16, el comportamiento fue similar al obtenido en las 8 y 12 horas, con la única diferencia que en este caso la estructura que primero recibió el impacto de los flujos de aire del exterior fue SHM1 (Fig. 3c). Las velocidades medias de los patrones de flujo de aire en el interior de SHM1, MG y SHM2 presentaron valores de $0,83,0,86$ y $1,06 \mathrm{~m} / \mathrm{s}$, respectivamente, lo que representa una reducción respecto a la velocidad del viento en el exterior con 41,2, 42,7 y 52,7\% en cada caso. Los valores medios de velocidad para los otros escenarios simulados se observan en el Cuadro 4.

Distribución espacial de la temperatura y la humedad relativa: Las distribuciones espaciales de la temperatura en el perfil horizontal a una altura de $1,6 \mathrm{~m}$ sobre el nivel del suelo para las 8,12 y 16 horas mostraron un comportamiento homogéneo con diferencias entre los puntos más fríos y cálidos en el interior de SHM1, MG y SHM2 con valores inferiores a $1^{\circ} \mathrm{C}$ (Fig. 3a,3b,3c). Así mismo, las distribuciones de la humedad relativa en este perfil horizontal mostraron una diferencias entre los puntos de mayor y menor humedad en cada una de las estructuras con valores inferiores al $4 \%$ (Fig. 3d,3e,3f).

Por otro lado, el valor medio de la temperatura en el interior de las estructuras para cada uno de los escenarios evaluados se puede observar en el Cuadro 5. Para SHM1 los valores de temperatura encontrados variaron entre el rango de 28,3 y $33,9^{\circ} \mathrm{C}$, lo que, represento valores de diferencial térmico entre el ambiente exterior e interior de 0,4 y $0,8^{\circ} \mathrm{C}$, respectivamente.

En el caso de MG los valores medios de temperatura presentaron una variación entre 28,1 y $33,7^{\circ} \mathrm{C}$, lo cual, generó diferenciales térmicos de 0,2 y 0,6 en cada caso, por último, para $\mathrm{SHM} 2$ los valores medios de temperatura tuvieron una variación entre 28,0 y $33,5^{\circ} \mathrm{C}$ por lo cual los diferenciales térmicos presentaron valores de 0,1 y $0,4^{\circ} \mathrm{C}$. 


\section{CUADRO 4}

Valores medios de velocidad de los patrones de flujo de aire $\left(\mathrm{ms}^{-1}\right)$ para los escenarios simulados

\begin{tabular}{cccc}
\hline Hora & $\begin{array}{c}\text { SHM1 } \\
\text { Velocidad } \\
\text { media Interior } \\
(\mathbf{m} / \mathbf{s})\end{array}$ & $\begin{array}{c}\text { MG } \\
\text { Velocidad } \\
\text { media Interior } \\
(\mathbf{m} / \mathbf{s})\end{array}$ & $\begin{array}{c}\text { SHM2 } \\
\text { Velocidad media } \\
\text { Interior }(\mathbf{m} / \mathbf{s})\end{array}$ \\
\hline 8 & 0,97 & 0,87 & 1,34 \\
9 & 0,87 & 0,78 & 1,25 \\
10 & 0,99 & 0,88 & 1,32 \\
11 & 1,04 & 0,91 & 1,51 \\
12 & 1,10 & 1,01 & 1,92 \\
13 & 0,98 & 0,91 & 1,65 \\
14 & 0,96 & 0,93 & 1,01 \\
15 & 0,97 & 0,91 & 1,12 \\
16 & 0,83 & 0,86 & 1,06 \\
\hline
\end{tabular}

\section{CUADRO 5}

Valores medios de los parámetros térmicos evaluados en el interior de cada una de las estructuras.

\begin{tabular}{ccccccc}
\hline Hora & \multicolumn{2}{c}{ Estructura SHM1 } & \multicolumn{2}{c}{ Estructura MG } & \multicolumn{2}{c}{ Estructura SHM2 } \\
\cline { 2 - 6 } & $\begin{array}{c}\text { Temperatura } \\
\text { media } \\
\text { Interior }\left({ }^{\circ} \mathrm{C}\right)\end{array}$ & $\begin{array}{c}\text { Diferencial } \\
\text { térmico } \\
\left({ }^{\circ} \mathrm{C}\right)\end{array}$ & $\begin{array}{c}\text { Temperatura } \\
\text { media } \\
\text { Interior }\left({ }^{\circ} \mathrm{C}\right)\end{array}$ & $\begin{array}{c}\text { Diferencial } \\
\text { térmico } \\
\left({ }^{\circ} \mathrm{C}\right)\end{array}$ & $\begin{array}{c}\text { Temperatura } \\
\text { media } \\
\text { Interior }\left({ }^{\circ} \mathrm{C}\right)\end{array}$ & $\begin{array}{c}\text { Diferencial } \\
\text { térmico } \\
\left({ }^{\circ} \mathrm{C}\right)\end{array}$ \\
\hline 8 & 28.3 & 0,4 & 28,1 & 0,2 & 28,0 & 0,1 \\
9 & 28,6 & 0,5 & 28,4 & 0,3 & 28,3 & 0,2 \\
10 & 29,9 & 0,5 & 29,7 & 0,3 & 29,6 & 0,2 \\
11 & 31,2 & 0,7 & 30,9 & 0,4 & 30,8 & 0,3 \\
12 & 31,7 & 0,4 & 31,6 & 0,3 & 31,5 & 0,2 \\
13 & 33,9 & 0,8 & 33,7 & 0,6 & 33,5 & 0,4 \\
14 & 33,3 & 0,7 & 33,0 & 0,4 & 32,9 & 0,3 \\
15 & 32,2 & 0,4 & 32,3 & 0,5 & 32,5 & 0,7 \\
16 & 31,1 & 0,2 & 31,2 & 0,2 & 31,4 & 0,5 \\
\hline
\end{tabular}

En el caso de la humedad relativa, los valores medios obtenidos para cada simulación en el interior de las tres estructuras pueden observarse en el Cuadro 6 . El comportamiento de la humedad relativa mostró valores de 50,6 y $67,4 \%$ para SHM1, 53,2 y $68,2 \%$ para MG y 55,6 y $69,0 \%$ para SHM2, respectivamente.

\section{CUADRO 6}

Valores medios de la humedad relativa del aire en el interior de cada una de las estructuras.

\begin{tabular}{cccc}
\hline \multirow{2}{*}{ Hora } & \multicolumn{3}{c}{ Humedad relativa media Interior (\%) } \\
\cline { 2 - 4 } & SHM1 & MG & SHM2 \\
\hline 8 & 67,4 & 68,2 & 69,0 \\
9 & 63,2 & 66,9 & 67,5 \\
10 & 60,4 & 63,8 & 64,1 \\
11 & 56,3 & 58,1 & 59,7 \\
12 & 54,2 & 55,2 & 56,4 \\
13 & 51,3 & 53,2 & 55,6 \\
14 & 50,6 & 53,6 & 55,7 \\
15 & 55,7 & 59,8 & 58,5 \\
16 & 63,7 & 62,9 & 61,4 \\
\hline
\end{tabular}


a)
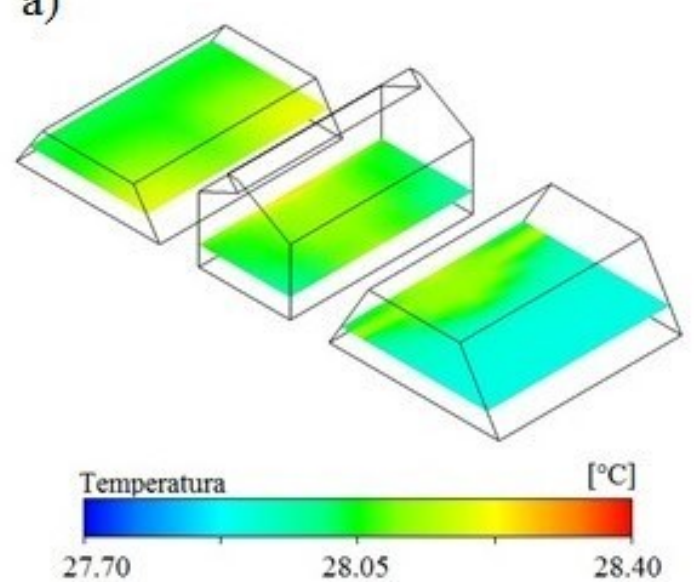

b)
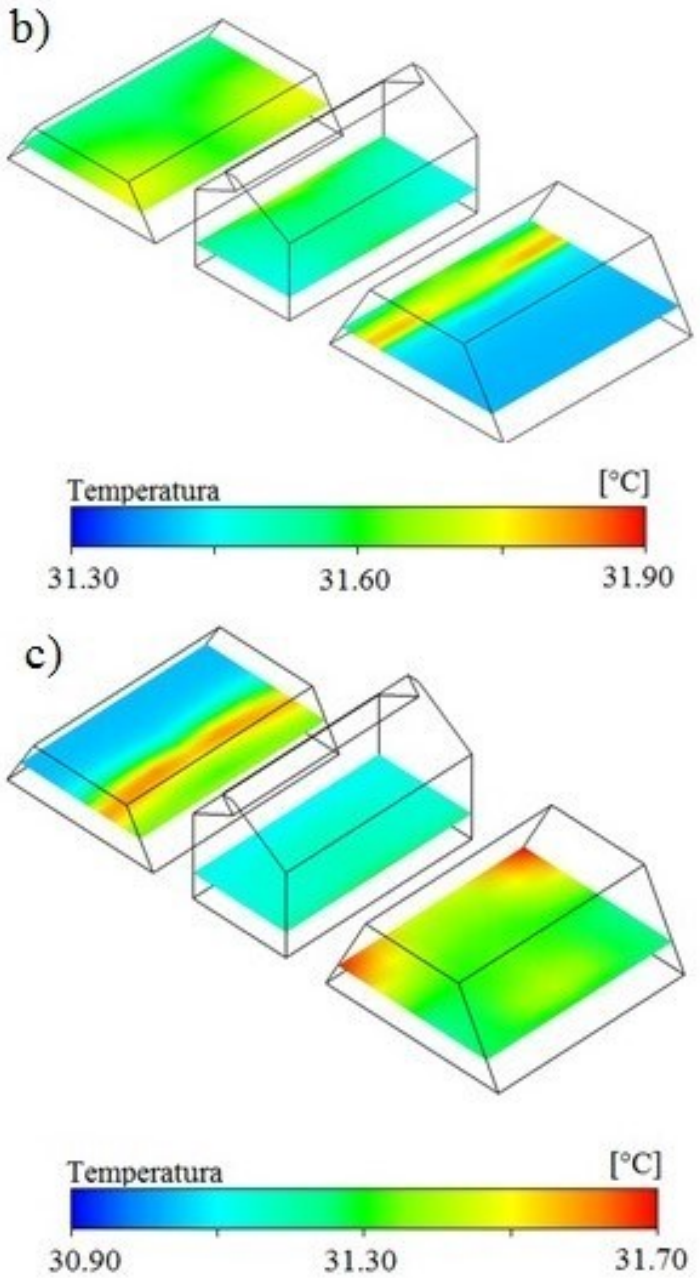
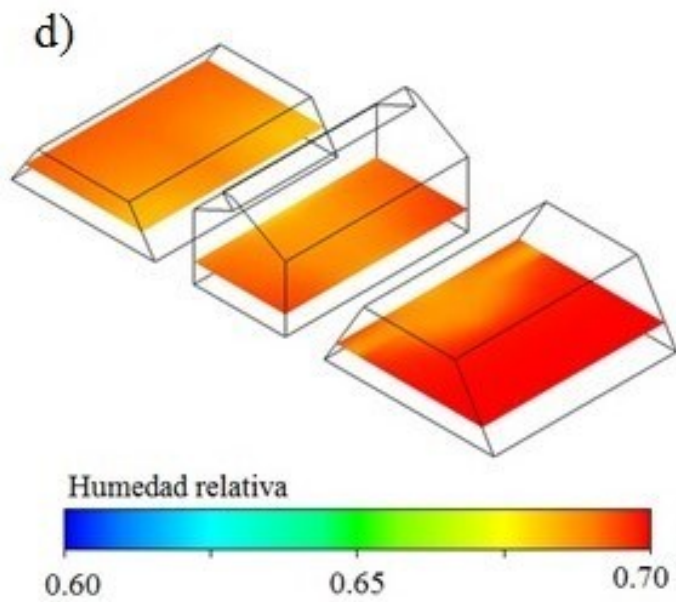

e)
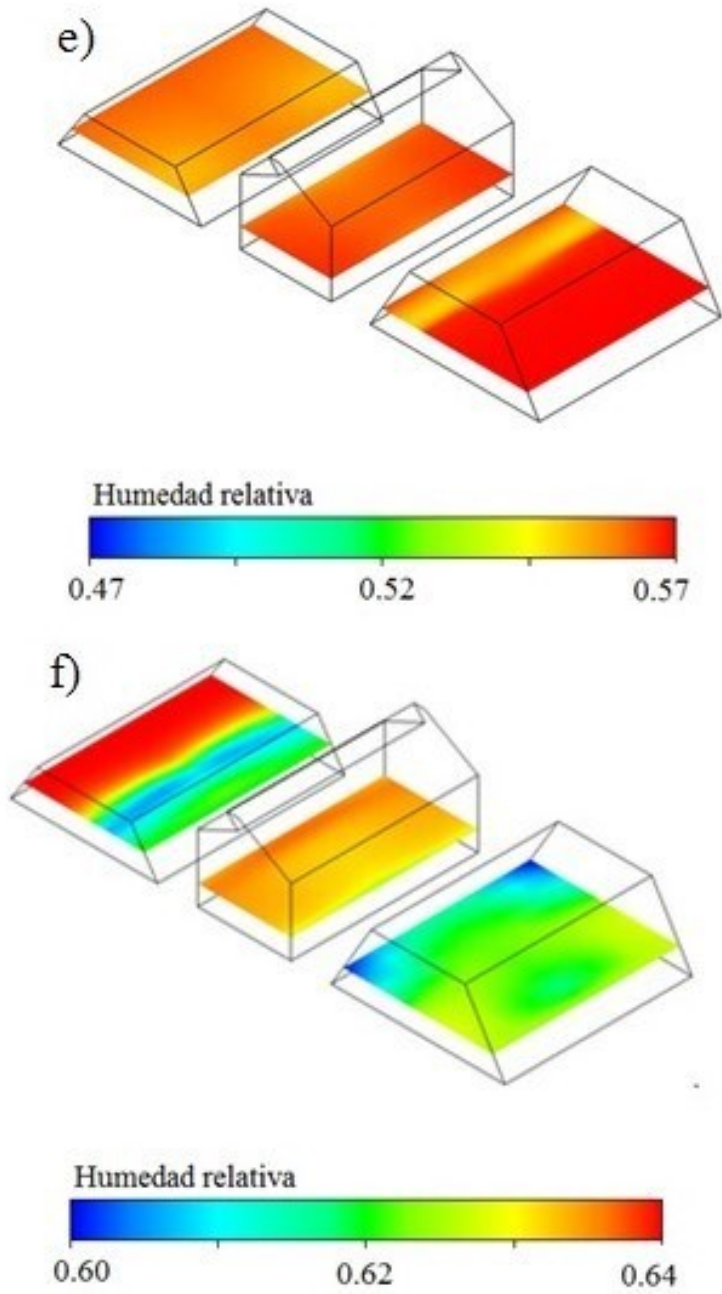

Fig. 4. Patrones de distribución térmica simulados $\left({ }^{\circ} \mathrm{C}\right)$, (a) Hora 8, (b) Hora 12, (c) Hora 16 y patrones de distribución espacial humedad relativa (\%) (d) Hora 8, (e) Hora 12 y (f) Hora 16. 


\section{DISCUSIÓN}

Validación del modelo CFD: Los Resultados obtenidos para la validación del modelo numérico pueden considerarse adecuados para este tipo de estudios, los valores de bondad de ajuste MAE y RMSE obtenidos entre los datos simulados y medidos para la temperatura fueron inferiores a $1^{\circ} \mathrm{C}$, estos valores están dentro del rango reportado en estudios previos realizados por otros autores (Majdoubi, Boulard, Fatnassi, \& Bouirden, 2009; Teitel, Dvorkin, Haim, Tanny, \& Seginer, 2009).

Así mismo para la variable humedad relativa los valores de MAE y RMSE fueron inferiores al valor de error máximo recomendado por Baptista, Bailey y Meneses (2012), quienes establecieron que un valor máximo de error del $10 \%$ puede considerarse como adecuado para esta variable cuando se usan modelos de simulación microclimatica para estructuras de agricultura protegida.

Por lo tanto, bajo estas consideraciones podemos concluir que el modelo numérico CFD 3D, predijo de manera satisfactoria las variables evaluadas durante el periodo y época de la evaluación experimental. Se debe resaltar que, de acuerdo con las tendencias de investigación a nivel mundial, este modelo numérico puede ser aplicado también para realizar el análisis micro climático de las estructuras evaluadas bajo otras condiciones climáticas del año o de otra región de Costa Rica, esto es posible puesto que una de las ventajas de los modelos CFD validados es su aplicabilidad con resultados precisos en escenarios no ocurridos o construidos (Alí, Bournet, Cannavo, \& Chantoiseau, 2018).

Patrones de flujo de aire: En general existe un patrón de movimiento de flujo que esta influenciado por la dirección del aire exterior y por la geometría de la estructura, donde los flujos de aire se desplazan desde los costados de barlovento hacia los costados de sotavento. Así mismo existe una característica en común y es la formación de una célula convergente entre las zonas de techo y el suelo del volumen de aire encerrado en la zona media de cada una de las estructuras. Estos resultados coinciden con los reportados por Teitel et al. (2015) y Flores-Velazquez y Montero (2008) para estructuras de casas de malla y reportados por Mesmoudi, Meguallati y Bournet (2017) para invernaderos.

Los valores de velocidad media de los patrones de flujo de aire en el interior de SHM1, MG y SHM2 para cada hora evaluada, permiten comprobar que existe una reducción general de la velocidad del flujo de aire, lo cual está influenciado por la presencia de pantallas anti-insectos (Valera, Álvarez, \& Molina, 2006). Este tipo de pantallas generan una pérdida de presión e impulso del flujo de aire, lo cual, se ve reflejado en la velocidad y movimiento del aire en el interior de las estructuras.

Los valores de pérdida de velocidad media calculados para los patrones de flujo de aire en el interior de las estructuras en relación con la velocidad del viento en el exterior para las horas evaluadas presento valores entre $27 \%$ y $64 \%$. Resultados que se encuentran dentro de los rangos reportados en estudios previos como los desarrollados por Molina-Aiz, Valera y López (2011) y Baeza et al. (2009).

Distribución espacial de la temperatura y humedad relativa: En estructuras de agricultura protegida uno de los factores más estudiados es la distribución espacial del microclima. Es de vital importancia analizar variables como; la temperatura y la humedad relativa del ambiente interior, ya que estas variables afectan los procesos de transpiración y fotosíntesis de las plantas, procesos relacionados directamente con el crecimiento y desarrollo de los cultivos y por ende con la productividad final de los mismos (Ma et al., 2019).

Los resultados obtenidos en este trabajo de investigación mostraron que existe una relación directa entre la velocidad y dirección del viento en el ambiente exterior y las distribuciones generadas de temperatura y humedad relativa en el interior de cada una de las estructuras. Por lo 
tanto, las áreas en el interior de las estructuras con mayor velocidad de flujo de aire presentan a su vez el menor valor de temperatura y el valor mayor de humedad, esto concuerda con el resultado obtenidos en estudios desarrollados para invernaderos y casas de malla (Flores-Velazquez \& Montero, 2008; Mesmoudi et al., 2017).

Las distribuciones espaciales de temperatura y humedad relativa sobre la zona de cultivo en cada una de las estructuras estudiadas mostraron un comportamiento que se puede considerar homogéneo, ya que las diferencias entre los valores máximos y mínimos para cada hora simulada fueron mínimos. Lo anterior se concluye al comparar los resultados de esta investigación con los resultados obtenidos en otros estudios de invernaderos y casa de malla donde para un momento de tiempo específico se reportan diferencias entre zonas en el interior de las estructuras con valores superiores a los $4^{\circ} \mathrm{C}$ para la temperatura y $15 \%$ para la humedad relativa (Flores-Velazquez, Guerrero, López, Montero, \& Piscia, 2013; Mesmoudi et al., 2017; Villagrán \& Bojacá, 2019b).

De acuerdo con los resultados de esta investigación podemos concluir que el modelo CFD mostró ser una herramienta efectiva para el estudio y análisis de los patrones de flujo, la distribución espacial de temperatura y humedad relativa en el interior de las estructuras de agricultura protegida bajo las condiciones climáticas de la temporada seca en Guanacaste, Costa Rica. Por lo tanto, la metodología usada en este trabajo podría aplicarse a investigaciones futuras en esta área del conocimiento y que tengan como objetivo evaluar un periodo de tiempo más largo donde puedan validarse y analizarse las condiciones climáticas dominantes que se presentan en las temporadas; húmeda y seca de la región.

Así mismo, se puede mencionar que en estructuras de tamaño y área reducida como las de esta investigación, la distribución del microclima no presento variaciones significativas entre los tres tipos de estructuras evaluadas. En este sentido, es recomendable que trabajos futuros se enfoquen en validar el comportamiento en estructuras de mayor área cubierta, donde es posible que si se puedan identificar diferencias significativas en el comportamiento del microclima.

\section{AGRADECIMIENTOS}

Los autores agradecen a la Corporación Colombiana de Investigación Agropecuaria AGROSAVIA y al Instituto Nacional de Innovación y Transferencia en Tecnología Agropecuaria (INTA) de Costa Rica por el apoyo técnico y administrativo en este estudio y al Fondo Regional de Tecnología Agropecuaria (FONTAGRO) por la financiación de la investigación denominada Innovaciones para la horticultura en ambientes protegidos en zonas tropicales: opción de intensificación sostenible de la agricultura familiar en el contexto de cambio climático en ALC. Las opiniones expresadas en esta publicación son únicamente las de los autores y no reflejan necesariamente los puntos de vista de FONTAGRO, su Consejo de Administración, el Banco, sus instituciones patrocinadoras o los países que representa. 


\section{ÉTICA, CONFLICTO DE INTERESES Y DECLARACIÓN DE FINANCIAMIENTO}

Los autores declaran haber cumplido con todos los requisitos éticos y legales pertinentes, tanto durante el estudio como en el manuscrito; que no hay conflictos de interés de ningún tipo, y que todas las fuentes financieras se detallan plena y claramente en la sección de agradecimientos. Asimismo, están de acuerdo con la versión editada final del documento. El respectivo documento legal firmado se encuentra en los archivos de la revista.

La declaración de la contribución de cada autor al manuscrito es la siguiente: E.A.V.M.:

Formulación del proyecto, planteamiento de la idea, escritura de las secciones de materiales y métodos, resultados y análisis y discusión, J.E.J.N.: Escritura y revisión de la sección de resultados y análisis de estos. Así mismo revisión y corrección del manuscrito final, R.I.L.P.: Formulación del proyecto, revisión de la metodología y participación en la escritura del documento en la sección metodológica y sección de resultados y discusión, y R.R.M.: Desarrollo de pruebas experimentales de campo y escritura del estado del arte incluido en la introducción del artículo y escritura de la sección de resultados.

\section{REFERENCIAS}

Ansys. (2016). ANSYS ICEM CFD User Manual. In Knowledge Creation Diffusion Utilization (Vol. 15317, Issue October). DOI: 10.1016/j.joen.2015.02.033.

Ali, H. B., Bournet, P. E., Cannavo, P., \& Chantoiseau, E. (2018). Development of a CFD crop submodel for simulating microclimate and transpiration of ornamental plants grown in a greenhouse under water restriction. Computers and Electronics in Agriculture, 149, 26-40. DOI: 10.17660/ActaHortic.2018.1227.5.

Baeza, E. J., Pérez-Parra, J. J., Montero, J. I., Bailey, B. J., López, J. C., \& Gázquez, J. C. (2009). Analysis of the role of sidewall vents on buoyancy-driven natural ventilation in parral-type greenhouses with and without insect screens using computational fluid dynamics. Biosystems Engineering, 104(1), 86-96. DOI: 10.1016/J.BIOSYSTEMSENG.2009.04.008.

Bañuelos-Ruedas, F., Ángeles-Camacho, C., \& Rios-Marcuello, S. (2010). Analysis and validation of the methodology used in the extrapolation of wind speed data at different heights. Renewable and Sustainable Energy Reviews,14(8), 2383-2391. DOI: 10.1016/j.rser.2010.05.001.

Baptista, F. J., Bailey, B. J., \& Meneses, J. F. (2012). Effect of nocturnal ventilation on the occurrence of Botrytis cinerea in Mediterranean unheated tomato greenhouses. Crop Protection, 32, 144-149. DOI: 10.1016/j.cropro.2011.11.005.

Bartzanas, T., Katsoulas, N., \& Kittas, C. (2012). Solar radiation distribution in screenhouses: A CFD approach. Acta Horticulturae, 956, 449-456. DOI: 10.17660/ActaHortic.2012.956.52

Espinal-Montes, V., Lorenzo López-Cruz, I., Rojano-Aguilar, A., Romantchik-Kriuchova, E., \& Ramírez-Arias, A. (2015). Determination of night-time thermal gradients in a greenhouse using computational thermal dynamics. Agrociencia, 49, 233-247.

Espinoza, K., López, A., Valera, D. L., Molina-Aiz, F. D., Torres, J. A., \& Peña, A. (2017). Effects of ventilator configuration on the flow pattern of a naturally-ventilated three-span Mediterranean greenhouse. Biosystems Engineering, 164, 13-30. DOI: 10.1016/j.biosystemseng.2017.10.001

Flores-Velazquez, J., \& Montero, J. I. (2008). Computational fluid dynamics (CFD) study of large scale screenhouses. Acta Horticulturae, 797, 117-122. DOI: 10.17660/ActaHortic.2008.797.14.

Flores-Velázquez, J., Mejía-Saenz, E., Montero-Camacho, J. I., \& Rojano, A. (2011). Analísis nuḿrico del clima interior en un invernadero de tres naves con ventilacín mećnica. Agrociencia, 45(5), 545-560. 
Flores-Velazquez, J., Guerrero, F. V., Lopez, I. L., Montero, J. I., \& Piscia, D. (2013). 3-Dimensional thermal analysis of a screenhouse with plane and multispan roof by using computational fluid dynamics (CFD). Acta Horticulturae, 1008, 151-158. DOI: 10.17660/ActaHortic.2013.1008.19.

Flores-Velazquez, J., Ojeda, W., Villarreal-Guerrero, F., \& Rojano, A. (2017). Effect of crops on natural ventilation in a screenhouse evaluated by CFD simulations. Acta Horticulturae, 1170, 95-101. DOI: 10.17660/ActaHortic.2017.1170.10.

He, X., Wang, J., Guo, S., Zhang, J., Wei, B., Sun, J., \& Shu, S. (2017). Ventilation optimization of solar greenhouse with removable back walls based on CFD. Computers and Electronics in Agriculture, 149,16-25 DOI: 10.1016/j.compag.2017.10.001

Kim, K., Yoon, J. Y., Kwon, H. J., Han, J. H., Eek Son, J., Nam, S. W., Giacomelli, G. A., \& Lee, I. B. (2008). 3-D CFD analysis of relative humidity distribution in greenhouse with a fog cooling system and refrigerative dehumidifiers. Biosystems Engineering, 100(2), 245-255. DOI: 10.1016/j.biosystemseng.2008.03.006

Ma, D., Carpenter, N., Maki, H., Rehman, T. U., Tuinstra, M. R., \& Jin, J. (2019). Greenhouse environment modeling and simulation for microclimate control. Computers and Electronics in Agriculture, 162, 134-142. DOI: 10.1016/j.compag.2019.04.013

MAG. (2018). Boletín del programa nacional sectorial de producción agrícola bajo ambientes protegidos. Recuperado de http://www.mag.go.cr/acerca_del_mag/estructura/oficinas/prog-nac-aprot.html.

Majdoubi, H., Boulard, T., Fatnassi, H., \& Bouirden, L. (2009). Airflow and microclimate patterns in a one-hectare Canary type greenhouse: an experimental and CFD assisted study. Agricultural and Forest Meteorology, 149(6-7), 10501062. DOI:10.1016/j.agrformet.2009.01.002.

McCartney, L., \& Lefsrud, M. G. (2018). Field trials of the Natural Ventilation Augmented Cooling (NVAC) greenhouse. Biosystems Engineering, 174, 159-172. DOI: 10.1016/j.biosystemseng.2018.07.004.

Mesmoudi, K., Meguallati, K., \& Bournet, P. (2017). Effect of the greenhouse design on the thermal behavior and microclimate distribution in greenhouses installed under semi-arid climate. Heat Transfer-Asian Research. DOI: 10.1002/htj.21274.

Molina-Aiz, F. D., Valera, D. L., Peña, A. A., Gil, J. A., \& López, A. (2009). A study of natural ventilation in an Almería-type greenhouse with insect screens by means of tri-sonic anemometry. Biosystems Engineering, 104(2), 224-242. DOI: 10.1016/j.biosystemseng.2009.06.013.

Molina-Aiz, F. D., Valera, D. L., \& López, A. (2011). Airflow at the openings of a naturally ventilated Almería-type greenhouse with insect-proof screens. Acta Horticulturae, 893, 545-552. DOI: 10.17660/ActaHortic.2011.893.56.

Molina-Aiz, F. D., Norton, T., López, A., Reyes-Rosas, A., Moreno, M. A., Marín, P., Espinoza, K., \& Valera, D. L. (2017). Using computational fluid dynamics to analyse the $\mathrm{CO} 2$ transfer in naturally ventilated greenhouses. Acta Horticulturae, 1182, 283-292. DOI:10.17660/ActaHortic.2017.1182.34

Norton, T., Sun, D.-W., Grant, J., Fallon, R., \& Dodd, V. (2007). Applications of computational fluid dynamics (CFD) in the modelling and design of ventilation systems in the agricultural industry: A review. Bioresource Technology, 98(12), 2386-2414. DOI: 10.1016/j.biortech.2006.11.025.

Perén, J. I., van Hooff, T., Leite, B. C. C., \& Blocken, B. (2016). CFD simulation of wind-driven upward cross ventilation and its enhancement in long buildings: Impact of single-span versus double-span leeward sawtooth roof and opening ratio. Building and Environment, 96, 142-156. DOI: 10.1016/j.buildenv.2015.11.021.

Piscia, D., Muñoz, P., Panadès, C., \& Montero, J. I. (2015). A method of coupling CFD and energy balance simulations to study humidity control in unheated greenhouses. Computers and Electronics in Agriculture, 115, 129-141. DOI: 10.1016/J.COMPAG.2015.05.005. 
Ramírez-Vargas, C., \& Nienhuis, J. (2012). Evaluación del crecimiento y productividad del tomate (Lycopersicon esculentum Mill) bajo cultivo protegido en tres localidades de Costa Rica. Revista Tecnología En Marcha, 25(1), 3-15.

Rojas Rishor, A. (2015). Análisis del comportamiento térmico de un invernadero construido en ladera, aplicando dinámica de fluidos computacional. (Tesis de pregrado, Universidad De Costa Rica, San José, Costa Rica). Recuperado de http://repositorio.sibdi.ucr.ac.cr:8080/jspui/bitstream/123456789/2946/1/38803.pdf

Saberian, A., \& Sajadiye, S. M. (2019). The effect of dynamic solar heat load on the greenhouse microclimate using CFD simulation. Renewable Energy, 138, 722-737 DOI: 10.1016/j.renene.2019.01.108.

Teitel, M., Dvorkin, D., Haim, Y., Tanny, J., \& Seginer, I. (2009). Comparison of measured and simulated flow through screens: Effects of screen inclination and porosity. Biosystems Engineering, 104(3), 404-416. DOI: 10.1016/j.biosystemseng.2009.07.006.

Teitel, M., \& Wenger, E. (2014). Air exchange and ventilation efficiencies of a monospan greenhouse with one inflow and one outflow through longitudinal side openings. Biosystems Engineering, 119, 98-107. DOI: 10.1016/j.biosystemseng.2013.11.001.

Teitel, M., Garcia-Teruel, M., Ibanez, P. F., Tanny, J., Laufer, S., Levi, A., \& Antler, A. (2015). Airflow characteristics and patterns in screenhouses covered with fine-mesh screens with either roof or roof and side ventilation. Biosystems Engineering, 131, 1-14. DOI: 10.1016/j.biosystemseng.2014.12.010

Tominaga, Y., Mochida, A., Yoshie, R., Kataoka, H., Nozu, T., Yoshikawa, M., \& Shirasawa, T. (2008). AlJ guidelines for practical applications of CFD to pedestrian wind environment around buildings. Journal of Wind Engineering and Industrial Aerodynamics, 96(10-11), 1749-1761. DOI: 10.1016/j.jweia.2008.02.058.

Tong, G., \& Christopher, D. M. (2018). New insights on span selection for Chinese solar greenhouses using CFD analyses. Computers and Electronics in Agriculture, 149, 3-15. DOI: 10.1016/J.COMPAG.2017.09.031.

Tong, G., Christopher, D. M., \& Zhang, G. (2018). New insights on span selection for Chinese solar greenhouses using CFD analyses. Computers and Electronics in Agriculture, 149, 3-15. DOI: 10.1016/j.compag.2017.09.031.

Valera, D. L., Álvarez, A. J., \& Molina, F. D. (2006). Aerodynamic analysis of several insect-proof screens used in greenhouses. Spanish Journal of Agricultural Research, 4(4), 273-279. DOI: 10.5424/sjar/2006044-204.

Villagrán, E.A., Gil, R., Acuña, J. F., \& Bojacá, C. R. (2012). Optimization of ventilation and its effect on the microclimate of a colombian multispan greenhouse. Agronomia Colombiana, 30(2), 282-288.

Villagran, E., \& Bojaca, C. (2019a). CFD Simulation of the Increase of the Roof Ventilation Area in a Traditional Colombian Greenhouse: Effect on Air Flow Patterns and Thermal Behavior. International Journal of Heat and Technology, 37(3), 881-892. DOI:10.18280/ijht.370326.

Villagrán, E, A., Baeza Romero, E. J., \& Bojacá, C. R. (2019). Transient CFD analysis of the natural ventilation of three types of greenhouses used for agricultural production in a tropical mountain climate. Biosystems Engineering. 188, 288304. DOI:10.1016/j.biosystemseng.2019.10.026.

Villagrán, E,A., \& Bojacá, C. R. (2019b). Study of natural ventilation in a Gothic multi-tunnel greenhouse designed to produce rose (Rosa spp.) in the high-Andean tropic. Ornamental Horticulture, 25(2), $133-143$. DOI:10.14295/oh.v25i2.2013.

Villagrán Munar, E. A., \& Bojacá Aldana, C. R. (2019). Simulacion del microclima en un invernadero usado para la producción de rosas bajo condiciones de clima intertropical. Chilean Journal of Agricultural \& Animal Sciences, 35(2), 137-150. DOI:10.4067/s0719-38902019005000308.

Villagrán, E., Ramirez, R., Rodriguez, A., Pacheco, R. L., \& Jaramillo, J. (2020). Simulation of the Thermal and Aerodynamic Behavior of an Established Screenhouse under Warm Tropical Climate Conditions: A Numerical Approach. International Journal of sustainable development and Planning, 15(4), 487-499. DOI: 10.18280/ijsdp.150409. 